\title{
Enseñanza universitaria y literacidades académicas: perspectivas presentes y horizontes futuros
}

\author{
Virginia Orlando ${ }^{1}$ \\ Facultad de Humanidades y Ciencias de la Educación, Universidad de la República, Montevideo, Uruguay
}

Resumen: Este artículo presenta una discusión sobre la participación de los procesos de lectura y escritura en el ámbito universitario. Trabajo desde una aproximación teórica que asume las prácticas de literacidad en clave sociocultural, en articulación con una epistemología dialogista de base bajtiniana. El contexto de investigación consiste en dos laboratorios de análisis de lectura y escritura en la educación superior desde un abordaje de contenidos específicos, ofrecidos en una universidad pública, y dirigidos a docentes pertenecientes a las distintas áreas del conocimiento de esa institución. A partir de tres "metaeventos" letrados acontecidos en esos talleres, revisados desde una "pedagogía de las multiliteracidades" (KALANTZIS y COPE 2005), surgen cuestiones que a mi entender deberían considerarse al proponer acciones didácticas que involucren la lectura y la escritura en distintos ámbitos disciplinares.

Palabras clave: Literacidades académicas; Enseñanza universitaria; Pedadogía de las multiliteracidaes.

Título: Ensino universitário e letramento acadêmico: perspectivas atuais e horizontes futuros

Resumo: Este artigo apresenta uma discussão sobre a participação dos processos de leitura e escrita no ambiente universitário. Trabalho a partir de uma abordagem teórica que pressupõe as práticas de letramento em chave sociocultural, em articulação com uma epistemologia dialogista de cunho bakhtiniano. $O$ contexto da pesquisa consiste em duas oficinas de análise de leitura e escrita no ensino superior, a partir de uma abordagem específica de conteúdos, oferecidas em uma universidade pública, e endereçadas a professores pertencentes às diferentes áreas de conhecimento daquela instituição. A partir de três "metaeventos" letrados desenvolvidos nessas oficinas e revisados a partir de uma "pedagogia dos multiletramentos" (KALANTZIS e COPE, 2005), surgem questões que a meu ver deveriam ser consideradas ao propor ações didáticas que envolvam leitura e escrita nos diferentes campos disciplinares.

Palavras-chave: Letramentos acadêmicos; Ensino universitário; Pedagogia dos multiletramentos.

Title: University Teaching and Academic Literacies: Present Perspectives and Future Horizons

Abstract: This paper presents a discussion about the participation of the reading and writing processes in the university environment. I work from a theoretical approach that assumes the practices of literacy in a sociocultural key, in articulation with a bakthinian dialogic epistemology. The research context consists of two workshops for reading and writing analysis in higher education from a specific content approach. Those workshops, offered at a public university, are aimed at teachers belonging to the different areas of knowledge

\footnotetext{
1 Doutora em Letras, Universidade Católica de Pelotas. Instituto de Lingüística, Facultad de Humanidades y Ciencias de la Educación. Orcid: https://orcid.org/0000-0002-8365-0206

E-mail: vir.orlando@gmail.com
} 
of that institution. From three "metaevents" occurred in these workshops, reviewed from a "pedagogy of multiliteracies" (KALANTZIS and COPE, 2005), arise questions that in my opinion should be considered when proposing didactic actions that involve reading and writing in different disciplinary fields.

Keywords: Academic literacies; Academic teaching; Multiliteracies pedagogy.

En este trabajo abordo algunos aspectos vinculados a los procesos de enseñanza y aprendizaje de la lectura y la escritura en el ámbito universitario. Mi relación con esta temática se sustenta no solo en intereses académicos que han orientado las investigaciones desarrolladas en los últimos años ${ }^{2}$, sino también en las actividades de enseñanza que desempeño como integrante de una institución universitaria y la reflexión desarrollada sobre tales actividades.

Diversos factores ${ }^{3}$ han hecho que en la última década la lectura y la escritura académica formen parte de la agenda sobre cuestiones a trabajar en lo atinente a las trayectorias académicas estudiantiles en el espacio de la universidad pública uruguaya. Entre las acciones institucionales implementadas recientemente se cuenta la oferta de laboratorios (es decir, talleres) de análisis de lectura y escritura en la educación superior desde un abordaje de contenidos específicos ${ }^{4}$. Estos laboratorios están dirigidos a profesores de las distintas áreas del conocimiento pertenecientes a esa universidad, con el objetivo, entre otros, de identificar y /o explicitar los problemas disciplinares en la escritura académica y reflexionar y discutir sobre estas cuestiones. Durante la oferta de la primera edición, consistente en una serie de encuentros quincenales a lo largo de un semestre, acompañé los encuentros como observadora participante y realicé su registro en soporte audio. ${ }^{5}$ En esta oportunidad reviso varios "metaeventos" de literacidad acontecidos en los laboratorios mencionados, desde una aproximación teórica que asume las prácticas de literacidad en clave sociocultural. A continuación, y antes de considerar esos "metaeventos", presento los elementos del abordaje teórico que orientan la discusión posterior.

\footnotetext{
${ }^{2}$ El planteo se enmarca en la línea de investigación sobre Prácticas letradas en ámbitos académicos, que forma parte de mi proyecto de Dedicación Total, financiado por la Comisión Sectorial de Investigación Científica de la Universidad de la República.

${ }^{3}$ Entre otras cosas, el aumento de la matrícula estudiantil en los estudios de grado y la valoración de establecer un acompañamiento a las trayectorias estudiantiles y a las formas de enseñanza universitarias.

${ }^{4}$ Se trata de la oferta de talleres enmarcados en la línea de cursos de Desarrollo Pedagógico Docente implementados en los últimos tres años a iniciativa de la Comisión Sectorial de Enseñanza. Esta última es un órgano central cuya finalidad es coordinar, estimular y desarrollar la actividad de enseñanza, entre otras cosas, mediante el impulso a la formación docente (véase http://www.cse.udelar.edu.uy).

${ }^{5}$ En ambos talleres se solicitó un consentimiento a los participantes para el uso de los datos en actividades de investigación.
} 


\section{Leer y escribir. La mirada teórica}

La alusión a literacidades en el título de este trabajo remite a una visión en la cual las acciones de leer y escribir están siempre "situadas", esto es: para leer textos del tipo X de la manera $\mathrm{Y}$, debemos tener experiencias en ambientes donde los textos del tipo $\mathrm{X}$ se lean de la manera Y (GEE, 2005). Esta afirmación asume a la lectura como algo diferente a decodificar los textos en la búsqueda de desentrañar sentidos estables y únicos, así como a la escritura como algo diferente a "fijar" mediante un código un sentido, nuevamente, estable y único. La "situación" de los textos refiere no solo a la consideración de un contexto de producción y circulación del texto, sino, y sobre todo, al hecho de que al leer y escribir, nuestras acciones como lectores y escritores hacen sentido en algún espacio del mundo social. Es decir, las prácticas de lectura y escritura son prácticas sociales ${ }^{6}$.

Esta aproximación a las acciones de leer y escribir, que integran de pleno derecho el mundo de las acciones lingüístico-discursivas, está en consonancia con el abordaje bajtiniano a los fenómenos del discurso. En dicho abordaje se considera a los diversos campos de la actividad humana ligados al uso del lenguaje (BAJTín, [1952-53]-2002) - de ahí su impronta sociocultural- y a ese uso en alguna situación, es decir, concretado en los enunciados individuales, orales o escritos, producidos y en circulación en esos distintos campos de actividad. Como toda producción discursiva y en tanto que enunciados, los textos escritos son realizaciones discursivas individuales producidas en incontables instancias de enunciación, que se matizan y modulan de diversa forma (BAJTíN, [1959-61]-2002). Esta diversidad no está librada a decisiones meramente individuales, sino que, en función del ámbito o esfera social que enmarca a los procesos de enunciación, se van agrupando como tipos relativamente estables de enunciados en los denominados géneros discursivos (BAJTíN, [1952-3]-2002).

Un acercamiento orientador, a mi entender, para dar cuenta de esa diversidad de enunciados (sean orales, sean escritos) producidos y en circulación dentro de los variados géneros discursivos, es el propuesto por Gee (2005) al considerar su existencia en el seno de Discursos ${ }^{7}$ primarios (conformados en el seno de los grupos que participan de la socialización primaria de un individuo en un determinado entorno sociocultural) y Discursos secundarios (elaborados mediante aprendizajes del individuo como parte de sus socializaciones en

\footnotetext{
${ }^{6}$ Los Nuevos Estudios sobre Literacidad (NLS), corriente que orienta este trabajo, no desconocen o reniegan de la dimensión psicológica involucrada en las literacidades (cf., entre otros, BARTON, 1994; STREET, 1993 y 2003). En este trabajo se enfatiza en los aspectos sociales por su pertinencia para la discusión posterior.

${ }^{7}$ Se entiende por Discurso "una asociación socialmente aceptada de formas de utilizar el lenguaje, otras expresiones simbólicas y "artefactos", de pensar, sentir, creer, valorar y actuar que puedan utilizarse para identificarse uno mismo como miembro de un grupo socialmente significativo o "red social", o para indicar (que uno está desempeñando) un "papel" socialmente significativo" (GEE, 2005: 144).
} 
distintos grupos e instituciones). Los Discursos secundarios apuntan a formas de socialización configuradoras de diversas identidades en variados ámbitos de la actividad humana (profesional, grupal, etc.).

Un segundo elemento orientador para la discusión que seguirá consiste en considerar al conocimiento letrado o literacidad como el dominio de un Discurso secundario que implica la letra (GEE, 2005: 156), y en consecuencia, el reconocimiento de múltiples literacidades, tantas como Discursos secundarios que convoquen la presencia de actividades letradas. Dado que el centro de atención en este trabajo es la enseñanza universitaria, en lo que respecta a ese ámbito aludiremos a literacidades académicas (o universitarias) ${ }^{8}$, relacionadas a diversos Discursos secundarios académicos y profesionales que configuran a su vez diversas identidades académico-profesionales.

Dejando de lado, en esta ocasión, la configuración identitaria que comenzaría a concretarse en el recorrido de la trayectoria curricular estudiantil, cabe preguntarnos sobre cómo se logra la apropiación de las literacidades académicas vinculadas a tales Discursos secundarios.

Si buscamos respuesta a esta cuestión en la reflexión dialogista ${ }^{9}$, la primera que surge apunta a que nos apropiamos de los usos del lenguaje en contacto con ellos, en las situaciones en las que nos encontramos; puesto que, como se plantea en Volóshinov, [1929]2009:130, "Los individuos no reciben una lengua acabada, sino que ingresan en esta corriente de la comunicación discursiva o, más bien, su conciencia se realiza por primera vez únicamente en esta corriente". Dicho de otro modo, los sentidos nacen del encuentro entre enunciados y Discursos pasados y futuros, es decir de esta forma de diálogo que se concretará de múltiples formas, según el campo de actividad del que se trate (SOBRAL, 2009).

En el caso de los usos del lenguaje que involucran algún conocimiento letrado, el abordaje en clave sociocultural a los procesos de familiarización en la lectura y escritura de los textos (en tanto que enunciados) asume que

la única manera de adquirir una determinada manera de leer cierto tipo de texto con "fluidez" o "propiedad" consiste en sumergirse (a modo de aprendiz), como miembro de una práctica social en la que las personas no

\footnotetext{
${ }^{8}$ Si bien podrían establecerse matices sobre el alcance del término "universitario" (que en español puede ser más abarcativo para referir a las variadas identidades, tanto académicas como profesionales) frente al término "académico", en este trabajo se emplearán las expresiones "literacidades universitarias" y "literacidades académicas" como sinónimas.

${ }^{9}$ La exégesis del aporte de Bajtín y su Círculo ha dado cuenta de la sofisticada elaboración que sustenta la noción de dialogismo (entre otros, BRAIT, 2006; FARACO, 2006), la cual puede condensarse en la siguiente reflexión del propio Bajtín ([1961]-2002: 334. El resaltado es mío.): “Vivir significa participar en un diálogo: significa interrogar, oír, responder, estar de acuerdo, etc. (...) El hombre se entrega todo a la palabra, y esta palabra forma parte de la tela dialógica de la vida humana, del simposio universal".
} 
solo lean esos textos de este tipo y de esta forma, sino que también hablen sobre esos textos de determinados modos, mantengan ciertas actitudes y valores sobre ellos e interactúen socialmente acerca de ellos de ciertas maneras (GEE, 2005: 56).

Ante la consideración previa, que parecería no admitir opciones ante la disyuntiva entre "adquirir" y "aprender", enfatizando la primera de las dos acciones, cabe insistir en el punto y buscar afinar su alcance. ¿Se trata, entonces, de adquirir los Discursos secundarios orientados por literacidades académicas mediante el contacto con modelos, procesos de ensayo y error y la práctica dentro de grupos sociales exclusivamente, sin una enseñanza formal, o también involucra el aprenderlos en procesos que implican un conocimiento logrado por medio de una enseñanza?

En líneas generales, el dominio de cualquier literacidad requiere el contacto con los modelos en ámbitos significativos y funcionales -es decir, su adquisición- en juego con un metaconocimiento -aprendido- (cf. GEE, op.cit.). En el caso específico de las literacidades académicas, adquisición y aprendizaje entran en juego, sobre todo a partir del "reclamo de transparencia institucional" que se instala en la tensión entre la problematización y visibilidad de los conocimientos letrados de los estudiantes frente a la frecuente invisibilidad de las literacidades disciplinares y de las prácticas pedagógicas en las que esas literacidades se despliegan, asumiéndose como "dadas" (LILLIS, 2001; cf. LEA y STREET, 1999).

La pregunta que me surge al reflexionar sobre el interjuego entre adquisión y aprendizaje de las literacidades académicas, es qué papel desempeñamos los profesores universitarios, si no en el aprendizaje, al menos en su enseñanza.

Podemos ensayar una respuesta desde la "pedagogía de las multiliteracidades" propuesta por Kalantzis y Cope (2005), y formulada en términos de cuatro elementos: práctica situada (que tenga en cuenta las experiencias previas de los aprendices), instrucción explícita (que permita elaborar a los aprendices un metalenguaje pertinente para elaborar sentidos propios, re-presentados y re-contextualizados), encuadre crítico (que relacione sentidos con contextos sociales y relaciones de valor de sistemas particulares de conocimiento y práctica social) y práctica transformadora (es decir, poder volver a la práctica desde una perspectiva reflexiva). Como señalan oportunamente los autores, no se trata necesariamente de elementos innovadores per se $y$, además, cada uno de los cuatro considerados aisladamente ha recibido diversas críticas, sin embargo, operando en conjunto "son necesarios para una buena enseñanza, aunque no de manera rígida o secuencial" (KALANTZIS y COPE, 2005: 240).

La pertinencia de pensar en una aproximación a las literacidades académicas que tenga en cuenta de forma integrada a tales elementos se discute en los próximos apartados de este artículo. Para articular la discusión selecciono tres (meta) eventos de literacidad acontecidos en los talleres mencionados al inicio de este trabajo, pero antes de pasar a los eventos en concreto, cabe aclarar algunas características del contexto en el cual se desarrollan. 


\section{El contexto de investigación}

Desde hace ya varios años, en la universidad pública uruguaya se desarrollan diversas acciones con foco en las literacidades académicas. Entre otras acciones se cuentan los talleres denominados Laboratorios de análisis de lectura y escritura en la educación superior desde un abordaje de contenidos específicos, ofrecidos por un conjunto de profesoras con formación específica en el área del lenguaje.

El primer año de la oferta participé de la discusión preparatoria de las actividades de los laboratorios asesorando sobre estas y sobre sus criterios orientadores. Se definió una convocatoria que agruparía a los participantes según las grandes áreas del conocimiento en las que se organiza la institución. Así, en esa instancia se propusieron dos talleres: uno congregaría docentes del área científico-tecnológica y del área de salud, mientras que el otro haría lo propio con docentes del área social y artística. El número de docentes inscriptos en el primer taller fue menor a diez, y en el segundo menor a treinta. Los participantes que acompañaron en su totalidad el primero fueron cinco, y quince el segundo. Luego, durante la oferta de ambos talleres desarrollados a lo largo de un semestre, cada uno de ellos a cargo de dos profesoras respectivamente ${ }^{10}$, acompañé los encuentros quincenales (de tres horas de duración cada uno) como observadora participante, al tiempo que los registré en soporte audio.

Como ya se dijo, la propuesta de los talleres buscaba, entre otras cosas, identificar y /o explicitar los problemas disciplinares en la escritura académica y reflexionar y discutir sobre estas cuestiones. El trabajo de cada encuentro estaba orientado por una pauta de las dos docentes a cargo que involucró la presentación de materiales en uso dentro de los cursos universitarios en los que los participantes del taller estaban trabajando, para su discusión en el taller.

En la medida que en las actividades desarrolladas a lo largo del semestre la lectura y la escritura - en este caso, académicas - desempeñaron un papel, podemos considerar los intercambios acontecidos desde la perspectiva de eventos letrados (cf. BARTON, 1994 y STREET, 1995). Todo evento letrado es una actividad particular y concreta que involucra la lectura y / o la escritura características de usos sociales específicos, es decir, de prácticas letradas que dan sentido a los usos de leer y escribir en situaciones específicas - en este caso, en la universidad (BARTON, 1994; GEE, 2000 y 2005; SOARES, 2006; STREET, 1995). Al mismo tiempo, dado el contexto en el cual se desarrollan los eventos letrados del taller, en múltiples ocasiones estos constituyeron el foco que orientó la discusión explícita acerca de las actividades letradas académicas. Precisamente por esta razón, aludiré a los eventos que presentan estas características como metaeventos letrados. En el próximo apartado presento tres metaeventos letrados acontecidos en los talleres para su discusión en lo concerniente a la presencia de la

\footnotetext{
${ }^{10}$ Se trata de dos docentes diferentes en cada uno de los dos talleres, que llevaron adelante una propuesta común y acordada entre las cuatro, cuestión que pude confirmar del acompañamiento que realicé de ambos talleres.
} 
lectura y la escritura en distintos ámbitos disciplinares y a cuestiones que podrían colaborar en la enseñanza universitaria, orientándome desde la "pedagogía de las multiliteracidades".

\section{Primer metaevento: ¿Manual A o manual B?}

Como expliqué antes, ciertas actividades del taller consistieron en el examen de materiales en uso dentro de los cursos universitarios en los que los participantes del taller estaban trabajando, para su discusión en el taller. En este caso, se trata de un metaevento que acontece en torno a las inquietudes manifestadas por una docente de una facultad perteneciente al área científico- tecnológica e integrante de un equipo docente responsable de la oferta de un curso sobre histología (curso $\mathrm{H}$ de aquí en más). Aludiré a esta docente como docente $\mathrm{H}$, así como docentes 1 y 2 a las docentes del taller (laboratorio) para mayor claridad en la exposición que sigue.

El compromiso del equipo docente del curso $\mathrm{H}$ incluye el cuidado por su índice de aprobación, cuidado previsible si tenemos en cuenta que en la vida universitaria el recorrido que todo individuo transita por la trayectoria curricular está acompañado permanentemente por instancias de evaluación de los conocimientos disciplinares que están construyendo durante el cursado de la carrera. Tales instancias, claves en lo que hace a la certificación de saberes, contribuyen a la retroalimentación de los procesos de aprendizaje y de enseñanza, puesto que las producciones -orales o escritas- del alumno ayudan al profesor a orientar el trabajo estudiantil futuro y a reflexionar sobre sus prácticas pedagógicas (CARLINO, 2007; cf. NARVAJA DE ARNOUX et al., 2006).

En las evaluaciones de la trayectoria inicial, el énfasis está puesto en la apropiación de un saber (disciplinar) que el estudiante debe hacer explícito en la producción de textos (escritos). Estos "encierran una doble complejidad: por un lado, deben adecuarse a las exigencias propias de la escritura académica; y por otro, deben evidenciar que se han realizado correctamente las operaciones de lectura demandadas por la institución" (NARVAJA DE ARNOUX et al., 2006:8). Es decir, los textos escritos en el contexto de evaluación académica involucran modalidades de lectura y de escritura propias de prácticas letradas académicas.

Al ser inicial en la carrera, el curso $\mathrm{H}$ cuenta con una matrícula estudiantil numerosa, cuestión que ha determinado paulatinamente la adopción de un formato de evaluación consistente en el planteo de preguntas de múltiple opción, sin la incorporación de preguntas u otras acciones que requieran desarrollo escrito en ningún caso. Sin embargo, si bien la numerosidad de la matrícula restringe la demanda de actividades escriturales en la evaluación del curso $\mathrm{H}$, las expectativas de lectura para su aprobación incluyen el acercamiento a textos sofisticados en su especificidad disciplinar. La docente $\mathrm{H}$ explica además que habría cierta dificultad en el acceso estudiantil al material bibliográfico, así como el uso recurrente (presente en otras carreras, según expresan en el taller otros participantes) y casi exclusivo de las 
presentaciones en Power Point que circulan en los cursos como material de preparación para los exámenes. Sin embargo, no es ese el único material existente, ya que si bien, como se dijo, habría alguna dificultad en el acceso a la bibliografía, se alude a dos manuales estructuradores del curso (de aquí en más MA y MB), y disponibles para los cursantes.

En el taller la docente $\mathrm{H}$ informa que en actividades de apoyo desarrolladas específicamente para estudiantes del curso $\mathrm{H}$ ha percibido que la lectura de los manuales plantea dificultades a los estudiantes, sin poder determinar en qué consisten específicamente las dificultades. Por esta razón las docentes 1 y 2 acuerdan con el grupo del taller destinar un tiempo del siguiente encuentro para revisar el abordaje realizado con un recorte temático (acerca de tejidos óseo, cartilaginoso y conjuntivo) desde ambos manuales y las actividades propuestas a los estudiantes del curso $\mathrm{H}$ a partir del material de lectura incluido en ese recorte temático.

Vale la pena acercarse al intercambio que se desarrolla a partir de la propuesta de trabajo de las docentes 1 y 2 teniendo en cuenta dos cosas. La primera refiere a la reflexividad sobre las acciones de lectura que dan el carácter "meta" al evento letrado que se desarrolla en ese intercambio surgido a partir de la propuesta de las docentes 1 y 2 con el grupo (de ahí, entonces, la denominación de metaevento letrado). La segunda consiste en aproximarse al intercambio desde los elementos integrantes de la pedagogía de las multiliteracidades referida previamente y que reitero ahora: práctica situada, instrucción explícita, encuadre crítico y práctica transformadora.

En el próximo encuentro del taller, las docentes 1 y 2, que han leído y trabajado sobre el material aportado por la docente $\mathrm{H}$, plantean la siguiente dinámica de trabajo. Distribuyen entre los participantes del taller una serie de preguntas vinculadas tanto al texto de MA como al de $M B$, mientras que a la docente $H$ le entregan una guía de preguntas sobre las actividades planteadas a propósito de los textos y sobre los textos en sí, provenientes de MA y MB, destinando un tiempo a la lectura individual y a la realización de la propuesta (aproximadamente veinte minutos), para pasar luego a una discusión en común (aproximadamente treinta minutos). Ese tiempo de trabajo individual posibilita a todos los participantes integrarse a la discusión desde el lugar del lector informado acerca de la realidad discursiva y la organización textual de MA y MB, aunque sean externos a la temática por no contar con la formación disciplinar específica. Por otra parte, permite a la docente $\mathrm{H}$ reflexionar acerca de los textos de ambos manuales, y de las actividades previstas en torno a ellos.

El intercambio desarrollado durante la discusión en común es muy extenso para poder presentarse en detalle, pero cabe detenerse en algunos de sus aspectos. Un hecho relevante destacado por las docentes 1 y 2 pasa por las propias diferencias existentes entre ambos manuales (cuestión sobre la que acuerdan todos los integrantes del taller). Según explica en ese intercambio la docente $\mathrm{H}$, el MA - que cuenta con años de publicado- si bien ha sido importante referencia entre los docentes de la especialidad, a entender de los especialistas ya estaría algo desactualizado. El MB, por su parte, es considerado ineludible para aportar un panorama completo y más actualizado. Sin embargo, a partir de la discusión dada en la puesta en común 
del grupo de taller, surgen elementos para valorar que el MB resulta más complejo para los estudiantes universitarios iniciales, sobre todo si no cuentan con lecturas previas sobre la temática específica. Veamos esos elementos con más detalle.

A partir de la lectura y de la reflexión acerca de la propuesta de trabajo para el taller, las docentes 1 y 2 comparten en la discusión con los participantes una serie de consideraciones, que en su conjunto pueden sintetizarse como sigue:

- el MA presenta el tema en un formato de capítulo que articula secuencias expositivas, descriptivas y explicativas, mientras que el MB se estructura básicamente en torno a secuencias descriptivas de mayor densidad terminológica;

- el MA recurre a recursos tipográficos y de otra índole siguiendo una estructuración "esperable" para un capítulo de libro, mientras que el MB recurre a una jerarquización menos obvia para el lector de los recursos tipográficos (en particular, un abundante uso del resaltado en negrita en combinación con dos tipos de fuente -y de tamaño- de letra distinta para los conceptos destacados) y una estructuración apegada casi exclusivamente a la presentación de párrafos descriptivos independientes entre sí. Estas características de MB pueden apreciarse en la Figura 1, presentada a modo ilustrativo en lo que hace a los aspectos tipográficos y a la disposición espacial dentro de la página.

Figura 1 - Presentación del Manual B

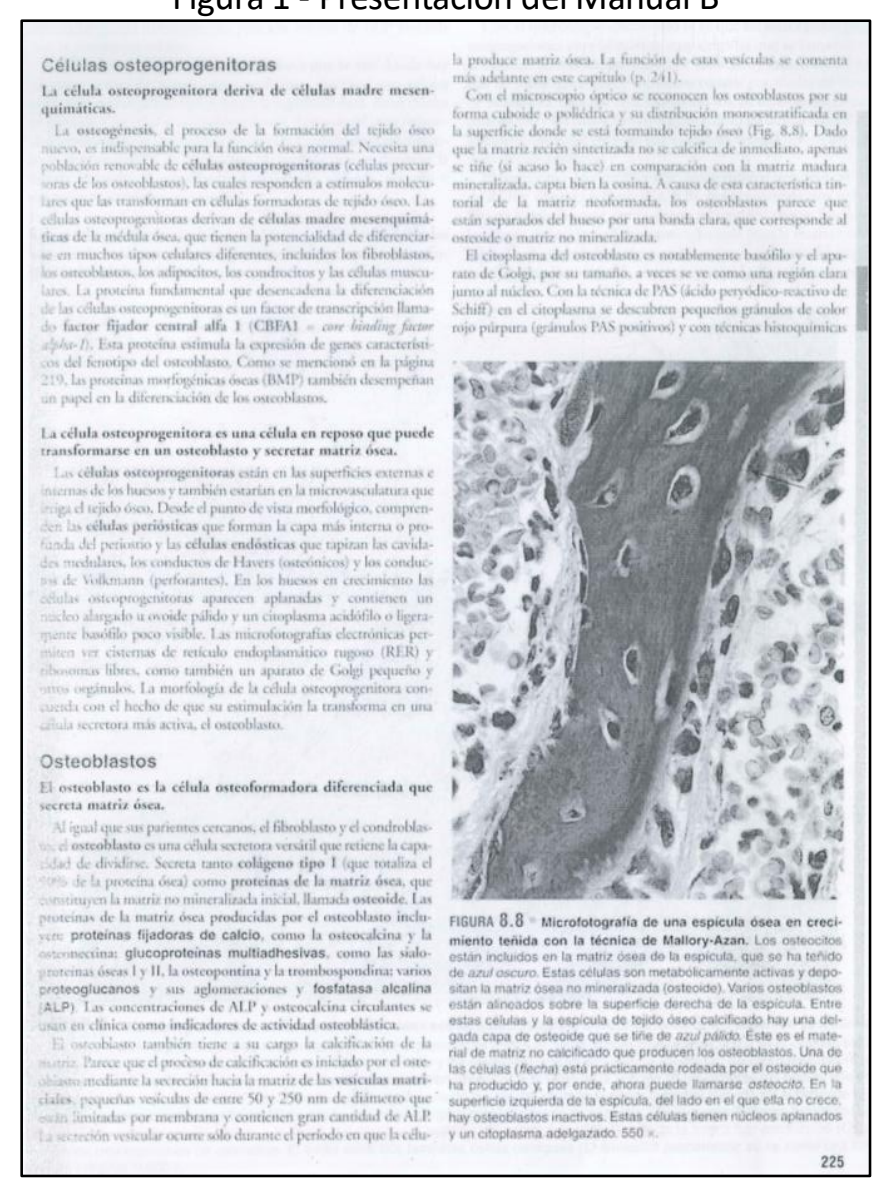

Fuente: ROSS, M. H.; PAWLINA, W. Histología: texto y atlas color con biología celular y molecular. Buenos Aires: Médica Panamericana, 2013. 
El punto que se pone en evidencia a partir de la mirada sobre estas características de MA y MB consiste en cómo la valoración de un material por características disciplinares no es lo único a considerar al plantear su lectura a estudiantes. Un texto muy recomendable por ciertas razones puede requerir especial atención al trabajo de lectura, por otras (razones).

Veamos un breve recorte del metaevento, relativo a estas cuestiones, en los minutos de cierre de la discusión.

Recorte del metaevento letrado: "La disyuntiva que tenemos todos"11

1. Docente 1- por eso yo te preguntaba cómo ustedes hacían dialogar los dos textos porque de repente es valioso que estén los dos.. nosotros no decimos que se descarten uno a otro (...) ((en $\mathrm{MB})$ ) uno va leyendo con otro detenimiento.. va incorporando los conceptos que importa que estén, y de repente llega en una segunda instancia a este otro texto, y si no hay mucha (...) discrepancia en lo que dice uno del otro, de repente hacerlos dialogar a favor del estudiante, o sea vincularlos explícitamente: este tema está así acá (...) yo los voy leyendo (...) si me falta un poco puedo acceder.. puedo leer primero por acá... después por acá

2. Docente $H$ - está bueno este planteamiento porque era, la disyuntiva que te digo, que tenemos todos...

3. OP-porque lo planteás como una disyunción.. y no como la posibilidad de..

4. Docente 2- de juntar

5. Docente $H$ - claro por eso.. porque en realidad.. como lo planteamos desde el rol del docente, habiendo leído los dos... a mí me pasa algo como lo que les pasó a ustedes.. yo capaz que como ya lo fui creando el MA.. ahora ya.. voy al otro ((al MB))...

6. Docente 2- claro... pero lo creaste!

7. Docente $H$ - lo tuve que hacer.. claro si me pongo a pensar.. el otro me permitió elaborar cosas.. que el otro.. (...) ya está resumido.. ya está como resumido.. aunque sea teniendo cosas claves pero para mí está como resaltado que en el otro... yo construí

(...)

8. $O P$ - (...) fíjate que también en la integración de los textos. no es menor el pensar cómo trabajan las imágenes en este tipo de textos... las leyendas que acompañan los textos... o sea el texto es todo... y sabemos que para ustedes... en especial por la disciplina.. el ojo importa mucho"

3er encuentro del Laboratorio 1

\footnotetext{
${ }^{11}$ Las convenciones manejadas para esta transcripción de la interacción discursiva, que no es de índole ortográfica, se aclaran a continuación. OP refiere a la observadora participante del taller, es decir, yo misma. Pausa media: .. Pausa larga: ... Discurso omitido: (...) Comentario o agregado de la analista: (()) Entonación exclamativa: !
} 
Varios puntos surgen del intercambio: en primer lugar, en el primer turno la docente 1 recupera un aspecto de la discusión que se ha desarrollado en los minutos previos, en la línea de poder "hacer dialogar" los textos antes que pensarlos en una suerte de antagonismo que lleve a que uno (MB) "descarte" (anule) a la lectura del otro (MA). Desde el recorrido de lectura que ha realizado esa docente con los textos de ambos manuales, su consideración -compartida por los participantes del taller luego de haberlos leído - es la posibilidad de contar con un primer acercamiento desde MA y trabajar integradamente con MB.

Por otra parte, la docente $\mathrm{H}$ comparte estas consideraciones (turnos 5 y 7). Cabe aclarar que mediante el recurso al verbo "crear" (presente en el turno 5, y retomado por la docente 2 en el turno 6) se recupera una metáfora que ha surgido en el intercambio previo, relativa a la apropiación individual de un conocimiento que en el texto perteneciente a MA es posible desde la progresión informativa y a su estructuración. También durante el intercambio previo, se ha examinado cómo el abordaje de los temas del curso desde MB resulta un aporte enriquecedor si se cuenta con una lectura previa de MA. En suma, la selección de textos no necesariamente implica una disyunción, sino la posibilidad de integración (OP y docente 2 en turnos 3 y 4 respectivamente). Vale la pena aclarar, asimismo, que la docente 1 ha insistido previamente con el cuidado que debe existir para una posible integración, relativo a acercamientos a la disciplina que pueden ser coincidentes en ciertos casos (el diálogo entre los textos se dará de una cierta forma en ese caso) y contradictorios en otros (y entonces el diálogo se establecerá desde la discrepancia de visiones o aproximaciones disciplinares).

Como ya dije, no solo la reflexividad acerca de las acciones de leer está en juego en este intercambio. Si recuperamos las dimensiones de la pedagogía de las multiliteracidades (práctica situada, instrucción explícita, encuadre crítico y práctica transformadora) para examinar el recorte de este metaevento, encontramos su participación doblemente: por una parte, en relación a los profesores universitarios en su carácter de participantes en el taller, y por otra, en relación con los estudiantes de los cursos universitarios en los que esos profesores participan.

En lo concerniente a los profesores como participantes del taller, es interesante observar cómo la docente $\mathrm{H}$ percibe su práctica (situada) de la lectura de ambos manuales en el turno 5: "como lo planteamos desde el rol del docente, habiendo leído los dos... a mí me pasa algo como lo que les pasó a ustedes", ubicándose primero en el lugar de profesora y descentrándose luego de ese rol al reconocerse próxima a los recorridos de lectura de las docentes 1 y 2 y de los participantes en el taller (en un papel cercano al de "lectores ingresantes a la disciplina" o si se quiere, de estudiantes de cursos universitarios iniciales). Así, en este intercambio de cierre del metaevento, vemos a la docente $\mathrm{H}$ saliendo de su lugar de lectora experta en la temática para considerar el camino de lectura desde la perspectiva de sus estudiantes. Ese descentramiento operado con la profesora podría operar luego en relación con los estudiantes de su curso.

Es importante señalar ahora que el alcance de la expresión práctica situada, cuando se plantea desde la pedagogía de las multiliteracidades, incorpora matices que van más allá de las 
consideraciones teóricas - presentadas al inicio de este trabajo - que orientan la visión de la lectura y la escritura como prácticas, precisamente, situadas. El sesgo que se imprime a la noción de "situación" pasa por considerar a las actividades de leer y escribir configuradoras de las literacidades, en este caso académicas, desde los diferentes roles y experiencias previas asumidos en una enseñanza particular y específica. Entonces, en el momento de plantear trabajos dentro de los cursos, el profesor "se sitúa" cuando calibra sus propuestas conjugando su experiencia letrada en la disciplina con la propia de los estudiantes ubicados en algún punto de la trayectoria curricular.

La posibilidad de "situarse" en la práctica abre las puertas a una forma de explicitación que podemos enmarcar, precisamente, en la dimensión de la instrucción explícita, otro de los elementos configuradores de esa pedagogía. En lo que hace al taller, las docentes 1 y 2 plantean a la docente $\mathrm{H}$ como trabajo para entregar en el siguiente encuentro una consigna de reformulación de actividades planteadas en torno a los textos de MA y MB que refiere, entre otras cosas, a "incluir consignas para trabajar con el vocabulario técnico y para hacer explícitas las relaciones entre los diferentes conceptos que se presentan en esos textos"12.

Al mismo tiempo, la explicitación de la docente $\mathrm{H}$ sobre la formulación de actividades para el curso $\mathrm{H}$ trae consigo la dimensión de la instrucción explícita con relación a los estudiantes de ese curso. Uno de los aportes de la docente 1 en el final del metaevento referido pasa por sugerirle a la docente $\mathrm{H}$ (que recepciona favorablemente lo sugerido) que en las actividades de apoyo a su curso puede abordar, en la línea de la propuesta de trabajo desarrollada ese día en el laboratorio, pasajes concretos de MA y MB para mostrar las diferentes presentaciones de ambos y presentar explícitamente a sus estudiantes los diferentes aportes que configuran la lectura de uno y otro. Acciones como esta, enmarcadas en la referida dimensión, permiten abordar las literacidades académicas desestructurando las "prácticas institucionales del misterio" (LILLIS, 2001) que enfrentan en muchas ocasiones a los estudiantes universitarios a leer y escribir según criterios propios de la comunidad disciplinar, muchas veces no dichos.

El rompimiento con las "prácticas misteriosas" cobra mayor pertinencia si se conjuga con un encuadre crítico (tercer elemento configurador de la pedagogía referida) de las literacidades académicas. Al respecto, es pertinente recuperar las palabras de Bazerman, dirigidas a estudiantes universitarios, que prologan un manual de escritura:

Es probable que, en tu vida escolar previa, vieras la escritura como una repetición de lo que decían los profesores y los textos leídos, para demostrar que habías aprendido y que podías transmitir de forma adecuada la información adquirida, usando la lengua correctamente. Sin embargo, ahora te están pidiendo algo más. Tus ideas, conocimiento, análisis y pensamiento crítico son fundamentales; pero deben construirse

\footnotetext{
${ }^{12}$ Tomado textualmente de la propuesta elaborada por las docentes 1 y 2 para $\mathrm{H}$.
} 
sobre la base del conocimiento acumulado por tu futuro campo profesional, con los modos de pensar, argumentar y presentar evidencia que le son propios (BAZERMAN, 2014: 11).

Tal como plantea el autor, los profesores universitarios demandamos "algo más" que una mera repetición de conocimientos de la lectura y la escritura académicas desarrolladas por nuestros estudiantes. Ese "algo más", según sigue el autor, se vincula con las formas específicas de pensar las problemáticas y las formas de actuar en el mundo según una determinada disciplina. El punto es que las formas específicas de las visiones disciplinares no están exentas de pugnas internas por el reconocimiento (y eventual imposición) de cierto cuerpo de conocimiento representativo (cf. HYLAND, 2004).

Debo reconocer que esta dimensión no está presente en el metaevento revisado, por lo que no profundizaré ahora en su desarrollo, aunque a lo largo del taller con esas mismas participantes emergerán ciertos elementos.

Para cerrar este apartado, corresponde preguntarnos en qué medida el trabajo desarrollado en el taller opera como práctica transformadora, en el caso del metaevento revisado, para la docente $H$ ( $y$, en el doble juego instalado inicialmente, para los estudiantes de su curso). Si por transformación entendemos la vuelta a la práctica desde una perspectiva reflexiva, el metaevento nos la presenta en el momento de "situarse" en la práctica, que finalmente es el punto de inflexión necesario para poder abordar reflexivamente sus prácticas posteriores. Sobre estas no hay acompañamiento realizado de mi parte que permita avanzar mayores consideraciones sobre sus acciones de enseñanza luego de finalizado el taller.

\section{Más metaeventos. Lo "no dicho" de las consignas}

En este apartado considero otros dos metaeventos. Mientras que en el presentado previamente el foco está en la selección de los textos, aquí se abordan, en primer lugar, las propuestas de actividades asociadas a la lectura de textos a trabajar dentro de los cursos universitarios, y en segundo lugar, la formulación de preguntas acerca de los textos.

El segundo metaevento también trata de la actividad desarrollada dentro del taller relativa a la presentación de materiales en uso dentro de los cursos universitarios en los que los participantes del taller estaban trabajando, para su discusión. Dicho metaevento acontece en torno al trabajo desarrollado en un curso sobre diseño (de aquí en más, curso D) dentro de una carrera perteneciente al área científico - tecnológica, sobre el cual explica una docente integrante del equipo a cargo (de aquí en más, docente $\mathrm{D}$ ). Al igual que con el metaevento revisado previamente, sus encargadas son las docentes 1 y 2 . El curso $D$ (con una matrícula estudiantil numerosa al igual que el curso $\mathrm{H}$ ) se propone introducir a los estudiantes en los debates teóricos contemporáneos sobre la disciplina, y a tales efectos se estructura en una 
alternancia de clases expositivas y clases de índole práctica, en las que se plantean muchas veces actividades asociadas a textos cuya lectura puede ser pautada domiciliariamente, antes de la clase, o durante la clase. Esos textos son luego tenidos en cuenta por el equipo docente en las pautas de evaluación para aprobación del curso, que incluyen respuestas de múltiple opción y también respuestas de elaboración breve.

En esta oportunidad, la docente $D$ presenta para su discusión una actividad prevista para las clases prácticas que incluye dos textos y las tareas que los estudiantes deben realizar en torno a ellos. De ambos textos, el segundo (de aquí en más texto 2) es un artículo breve de prensa disponible online, mientras que el primero (de aquí en más texto 1$)^{13}$ presenta otras características. Como ella misma señala, se trata de un texto de pertinencia en cuanto a su temática para el curso $\mathrm{D}$, sin mayores complejidades de índole terminológica, aunque de una extensión que supera notoriamente a la mayoría de los seleccionados para las actividades prácticas. Por otra parte, la docente $D$ se ha incorporado recientemente al equipo docente del curso, y la selección de los textos con los que trabaja no está a su cargo.

Al igual que con el primer metaevento, este se enmarca en la dinámica de trabajo que prevé la lectura del texto para los participantes del taller y una guía de preguntas sobre las actividades planteadas a propósito de los textos y los textos en sí para la docente D. La discusión en común, iniciada al final de uno de los encuentros del taller y retomada al siguiente, transcurre a lo largo de aproximadamente una hora. Focalizaré, con relación a ese intercambio, algunos aspectos vinculados a las consignas planteadas en torno a los textos. Como se señala en Natale y Stagnaro (2014), las consignas son instrumentos que median entre el docente y los estudiantes y entre lo solicitado como tarea y el texto a ser interpretado (en el caso de la lectura) o producido (en el caso de la escritura).

En la literatura especializada, es posible advertir consenso acerca de la función de la consigna. Así, se acepta su papel de orientadora y organizadora de la actividad de los estudiantes. La consigna expresa el tipo de demandas que realiza el docente durante las actividades de clase, y se presenta como el principal instrumento con que cuenta el profesor para orientar el esfuerzo cognitivo y las estrategias de aprendizaje de los alumnos [...]. Constituye un texto que configura las acciones mentales en los aprendices ya que en su formulación intenta regular, ordenar y dirigir las operaciones a realizar: define las acciones y estimula el tipo de actividades de lectura y escritura a realizar (VÁZQUEZ, 2007: 4-5).

En el caso de la actividad del curso $D$, las consignas que acompañan la lectura del texto son las siguientes: 1 . Lee el texto (texto 1 ), resume en el siguiente espacio ${ }^{14}$ de qué se trata. 2 .

\footnotetext{
${ }^{13}$ Cortina, A. "Quién, qué, por qué consumir".

Disponible en: https://antigua.solidaridad.net/noticia/1859/consumo-luego-existo-por-adela-cortina. Acceso en: 20/2/2019.

${ }^{14}$ Se incorpora en esa consigna un espacio acotado a cuatro líneas para desarrollar el resumen.
} 
Identifica tres palabras clave. 3. A partir del artículo (texto 2) identifica el problema y la solución planteada. 4. Argumenta a favor y en contra. 5. Expone en tres minutos a los demás compañeros.

Se podrían abordar diversos aspectos con relación a esas consignas ${ }^{15}$, pero aquí me ocuparé exclusivamente de aspectos relativos a la primera. Cabe iniciar señalando que las dos operaciones solicitadas en ellas con relación al texto 1 (leer y resumir), conllevan un tiempo de la discusión desarrollada en torno a las consignas de la actividad. En este caso, el recorte que se presenta a continuación forma parte del inicio de esa discusión, en relación con los propósitos buscados por los docentes del curso D mediante el planteo de la primera consigna.

Recorte del metaevento letrado: "Lo que se buscaba"

1. docente $D$-lo que se buscaba era.. una lectura del texto y de manera breve que contaran de que se trata en realidad eh cuando se pide de manera breve a veces es medio complicado porque.. tratar de resumir un texto tan amplio.. muchas veces se pierden conceptos es lo que al menos a mí me pasó (...) pero.. lo que yo venía pensando era que nosotros no practicamos antes las respuestas (...) es poder practicarnos las respuestas antes para ver qué es lo que puede suceder (...) cuando me entregaron la propuesta días antes.. yo realmente.. no me puse a pensar realmente qué era lo que podían contestar

2. docente 2-y también qué era lo que ustedes esperaban...

3. docente $D$ - claro.. lo que se espera en realidad es que haya un poder de síntesis.. no se espera una respuesta estructurada

4. docente 2- no eso entiendo.. pero qué puntos sí o sí deben estar

5. docente $D$ - deberían de ir

6. docente 2- en un resumen de este texto.. por ejemplo

7. docente $D$ - ahí está.. sí sí sí

8-docente 2- para después a ellos poderlos ayudar..

9- docente $D$ - sí para poder guiarlos

10. docente 2 -claro para eso hay que ensayarlo

11. docente $D$-por eso! ahí hay que ensayarlo y en muchas oportunidades hemos ensayado y nos hemos dado cuenta (...)

3er encuentro del Laboratorio 1

En este caso, cuando la docente D explica el propósito de la primera consigna,

${ }^{15}$ Una presentación detallada de este caso se encuentra en García de los Santos y Méndez Caulín (2018). 
concerniente a resumir a partir de la lectura del texto, señala igualmente que podría haber realizado las consignas antes de trabajar con los estudiantes: "venía pensando que nosotros no practicamos las respuestas antes para ver qué es lo que puede suceder" (turno 1), lo que sí ha hecho en otras ocasiones junto con el equipo docente: "en muchas oportunidades hemos ensayado y nos hemos dado cuenta" (turno 11).

Así, las referencias de la docente D a "poder practicarnos las respuestas" (en turno 1) y de ella y la docente 2 a "ensayarlo" (en turnos 10 y 11) metaforizan nuevamente el situarse en el lugar del estudiante al desarrollar las acciones solicitadas a ellos. Precisamente porque la docente $D$ explica que muchas veces sí lo hacen (turno 11) -y es ella misma quien se da cuenta que no lo ha hecho en esa ocasión - podemos ver que esa dimensión pedagógica de la literacidad está presente en la enseñanza desarrollada por este equipo docente, con relación al curso D.

Por otra parte, es en lo concerniente al grado de instrucción explícita vinculado a la concreción de las consignas que prosigue la discusión del taller, ya que como la propia docente D reconoce "es medio complicado (...) tratar de resumir un texto tan amplio" (turno 1). Precisamente acerca de las complejidades asociadas a la actividad de resumir, en especial cuando se trata de un texto no solo extenso sino también rico en su desarrollo conceptual, es que continúa la discusión en el taller. La propia docente $D$ afirma, minutos más adelante, que debería formularse la consigna de otra forma, si es que desde nuestras expectativas docentes buscamos que los estudiantes logren cumplir con ellas. En particular, la incorporación de "preguntas - guía" que operen, precisamente, como un apoyo en la jerarquización de ciertos puntos del texto, es uno de los posibles caminos sugeridos por las docentes 1 y 2 , en acuerdo con $\mathrm{D}$, en cuanto a ajustes en la instrucción explícita asociada a esa acción de resumir el texto, pautada en la consigna.

Para concluir la mirada acerca de este metaevento cabe agregar que en la discusión surgen aspectos relativos al encuadre crítico presente en la mirada pedagógica sobre los propósitos de las consignas del curso $D$, que no serán abordados en esta oportunidad. Sí es importante aclarar que la cuidadosa reformulación de las consignas realizada por la propia docente $D$ en el marco de las actividades del taller dan cuenta de su encaminamiento a una transformación de la práctica (que al igual que en el caso del metaevento previamente revisado, no puede precisarse al no haberse desarrollado un acompañamiento de las clases prácticas de la docente $\mathrm{D}$ con los estudiantes del curso D).

En cuanto al tercer y último metaevento a ser presentado aquí, este trata precisamente sobre la formulación de preguntas que los docentes planteamos vinculadas a los textos que damos para su lectura en los cursos universitarios. A diferencia de los metaeventos anteriores, que resultan del tratamiento de materiales proporcionados por los profesores participantes de los talleres en uso en sus propios cursos universitarios, este metaevento refiere a una actividad acordada por las cuatro profesoras encargadas de los dos talleres referidos al presentar el contexto de investigación. Asimismo, debe aclararse que en esta ocasión la mirada estará puesta en el taller integrado por profesores del área social y artística, a cargo de las docentes 3 y 
4 (de aquí en más).

El propósito del planteo apuntaba a mostrar la complejidad que involucra toda actividad de lectura y el planteo de preguntas asociadas, recurso empleado en la formulación de diversas consignas, ya sea a la hora de evaluar conocimientos, o de activar el destaque de ciertos conceptos y su recuperación a través de una lectura "guiada". Para el desarrollo de la actividad, se presentó un extracto (consistente en los tres primeros párrafos de la Introducción) de un artículo publicado en una revista especializada de lingüística, en el que se discute sobre la causativización en español, incorporado a continuación.

Cuadro 1 - Texto empleado en el tercer metaevento

\begin{abstract}
La causativización es un proceso gramatical ampliamente extendido en las lenguas que consiste en el agregado de un evento causativo a un predicado (Pylkkänen 2008). Sin embargo, las propiedades y distribución de las causativas presentan variación interlingüística considerable. Así, ciertas lenguas como el japonés permiten causativizar fácilmente todo tipo de verbos, pero otras como el inglés o el español hacen uso de diversos mecanismos dependiendo del tipo de predicado afectado por este proceso (véase la sección 4 en este trabajo).

Tradicionalmente, se reconocen dos tipos de causativas: las denominadas causativas sintéticas (también conocidas como causativas léxicas) (2a) y las causativas analíticas (o sintácticas) (2b):

1. Juan desapareció.

2. a. A Juan lo desaparecieron.

b. A Juan lo hicieron desaparecer.

Si bien la causativización analítica es un fenómeno muy estudiado en español (Bordelois 1974, 1988, Cano Aguilar 1977, Zubizarreta 1985, Alsina 1992, Treviño 1994, Ordóñez 2008, Torrego 2010 y Tubinio Blanco 2011, entre muchos otros), la sintética ha recibido poco a nula atención o incluso se ha negado su existencia[2]. De hecho, solo hay algunos trabajos que registran usos transitivos de verbos inacusativos (e.g., Demonte 2000, 2001, Makuc 2008 y la NGRALE 2009) o sancionan su uso como desviaciones dialectales de la norma (e.g., Cano Aguilar 1981). A nuestro leal saber y entender, solo en Zdrojewski (2007), Pujalte \& Zdrojewski (2007) y Pujalte (2012) y Pujalte \& Zdrojewski (2013) se discute este fenómeno desde una perspectiva descriptiva y teórica[3].
\end{abstract}

Fuente: PUJALTE, M. Estrategias de causativización en español. Lingüística, Vol. 29, n. 2, p. 231269, 2013.

Junto con el texto, para cuya lectura se destinaron algunos minutos, se formularon las siguientes preguntas: 1. ¿Qué es la causativización?, 2. ¿En qué lenguas se produce más fácilmente?, 3.¿Cuántos tipos de causativas hay?

En la puesta en común de la actividad, se desarrolla un intercambio de cuarenta minutos de duración aproximadamente, del cual solo se tomarán algunos elementos que sinteticen la discusión acontecida.

El intercambio inicia con la lectura de las respuestas elaboradas por los participantes del taller, que dada la índole de las preguntas, reproducen - correctamente- de forma casi literal el texto de partida ${ }^{16}$. Téngase en cuenta que ninguno de estos profesores universitarios cuenta

\footnotetext{
${ }^{16}$ Para responder la primera pregunta alcanzaba con reproducir el inicio del extracto distribuido: “La
} 
con conocimientos disciplinares propios de la temática del texto seleccionado: esto está buscado por las docentes del taller, precisamente para "situarlos" en el lugar del otro, es decir, del estudiante. Cuando están terminando con la lectura de las respuestas, acontece el recorte del metaevento siguiente.

Recorte del metaevento letrado: "Navegar sobre la superficie del texto"17

1. docente A- yo quiero hacer una mención sobre esto.. porque.. si yo soy la docente y me contestan esto en un escrito.. le voy a poner buena nota.. pero .. no es navegar sobre la superficie del texto?... quedó el concepto? (...) las preguntas me llevan a que mire la superficie del texto

2. docente $B$ - yo cuando dijiste que ibas a hacer unas preguntas dije... tá no voy a poder (--) porque el texto no lo entendí mucho pero en realidad las preguntas son (--)

3. docente 3- pero todo el mundo contestó! ((bromeando)) eras la única que no habías entendido! ((risas de todos y comentarios superpuestos))

20 encuentro del Laboratorio 2

La docente $\mathrm{A}$ expresa que las preguntas respondidas en la actividad propuesta llevan a "mirar la superficie del texto" (turno 1), mientras que la docente B agrega que "el texto no lo entendí mucho" (turno 2), afirmación que es acompañada por la broma de la docente 3 a cargo del taller señalando que "todo el mundo contestó" (turno 3). Cabe observar que luego de la broma, que sigue en la línea de: todos entendieron de qué se trata esto, y concita risas en el grupo en general (final del turno 3), se desarrolla una discusión cuyo punto de partida pasa por observar que todos pudieron dar una respuesta correcta, y sin embargo continúan desconociendo de qué se trata el fenómeno (en el texto propuesto, la causativización).

Así, el intercambio continúa en el debate entre, a decir de Scardamalia y Bereiter (1992), "decir el conocimiento" o "transformar el conocimiento"18: los participantes del taller

causativización es un proceso gramatical ampliamente extendido en las lenguas que consiste en el agregado de un evento causativo a un predicado."

${ }^{17} \mathrm{~A}$ las convenciones presentadas para la transcripción de la interacción discursiva, se agregan aquí otras. Por docente $A$ y docente $B$ se alude a docentes no mencionadas previamente, participantes del taller 2. Pasaje del discurso incomprensible: (--) Entonación interrogativa: ?

${ }^{18}$ Los autores refieren cada uno de esos modos a las operaciones psicológicas propias de los escritores novatos (que dependen para sus producciones de que el conocimiento se encuentre ya agrupado, sea en la memoria o en actividades propuestas por los profesores) y los experimentados (que procederán a una elaboración aunque el conocimiento no esté agrupado de forma coherente) respectivamente. "(...) lo que vemos en la obra de escritores experimentados, es la ejecución de procedimientos poderosos que les permiten delimitar, elaborar y refinar conocimientos disponibles. Para los novatos (...) la composición sirve, más bien, para reproducir que para refinar el 
concuerdan en que se puede responder un cuestionario (como el del ejercicio realizado por ellos) pero no entender. También con relación a este metaevento, el carácter situado de la práctica desde lo pedagógico, llevando a los participantes del taller a descentrarse de su lugar de profesores universitarios para ubicarse en el lugar de los estudiantes, orienta la discusión que sigue, que incorpora además las dimensiones relativas a la instrucción explícita y al encuadre crítico. A modo de síntesis de esa discusión, los dos ejes temáticos desarrollados problematizan el desafío que puede suponer (o no) la formulación de las preguntas sobre los textos, y las características de los textos académicos en sí mismos.

Sobre la formulación de las preguntas, surge en los planteos la importancia de calibrar situadamente su pertinencia, porque el carácter del cuestionario propuesto en el taller puede ser pertinente durante un curso como forma de "control de lectura" (en línea con lo discutido en el metaevento anterior, las preguntas pueden actuar como guía que resalte los conceptos asumidos como centrales o pertinentes de una determinada lectura enmarcada en un curso disciplinar), o ser inadecuado si buscamos promover la construcción de aprendizajes y evaluarlos. Finalmente, como señala un participante del taller, aprobar un curso no equivale a aprender (la disciplina).

En cuanto a los textos en sí mismos, la discusión se concentra en las características propias de textos académicos como el que orienta este metaevento, y en la construcción del conocimiento disciplinar que sustenta la organización de tales textos.

Por un lado, se discute sobre la pertinencia de trabajar explícitamente con los estudiantes universitarios - ya desde el inicio de las trayectorias curriculares - las convenciones técnicas que organizan la introducción de otras voces mediante la alusión al apellido de algún autor y el año en que ha producido su texto, así como la necesidad de ubicar (nuevamente, "situar") el fragmento presentado con relación a algún enunciador (¿quién ha escrito ese fragmento, las profesoras del taller? ¿alguien diferente?) y de ofrecer indicaciones sobre la existencia de notas - representadas como [2] y [3] en el pasaje seleccionado- que sin embargo no se han conservado en el texto presentado para realizar la actividad. Cabe señalar que son las profesoras del taller quienes han decidido plantear la actividad con la falta de referencia al autor, la supresión de las notas y la ausencia de las referencias bibliográficas del texto cuyo fragmento se ha examinado, buscando precisamente problematizar esas características de los textos académicos.

Por otro, tal como señala en el transcurso del metaevento la docente 3 , se trata de abordar "la incertidumbre en un lector inexperto que no conoce la materia", cuestión que va más allá de lo manifestado por otro participante del taller al señalar que en "la primera lectura [del fragmento] no entendí absolutamente nada porque... los términos mismos me resultaban absolutamente extraños". También ese efecto de incertidumbre o extrañamiento ha sido buscado con la propuesta de esta actividad del taller, y permite encaminar la reflexión de sus participantes en la línea del tejido dialógico que parafraseando a Bajtín, forma parte de la vida

conocimiento" (SCARDAMALIA y BEREITER, 1992: 63-64). 
académica. Así, si en ese fragmento se alude a "las causativas", ¿dónde está dicho en qué consisten? Como la docente 3 señala, la construcción del conocimiento académico resulta del conocimiento acumulado, que trae los "ecos de algo dicho antes", a lo que un participante del taller agrega que "es necesario que sea así porque en cada clase no podemos empezar de cero". Esta percepción dialógica se instala en el final del metaevento que concluye con la reflexión acerca de poder trabajar con consignas que trasciendan la reproducción literal y colaboren a ubicar esos aspectos dialógicos de todo texto - enunciado del mundo académico.

Como ya dije acerca de los metaeventos anteriores, la ausencia de un proceso de acompañamiento posterior a la realización del taller con los profesores no me permite dar cuenta de la dimensión transformadora de las prácticas en sus cursos universitarios. Sin embargo, me lleva a apuntar algunas reflexiones con las que finalizo este trabajo.

\section{Consideraciones de cierre. Qué nos dicen los metaeventos}

A partir de la mirada puesta en los metaeventos seleccionados, entiendo que parte de su riqueza está en desarrollar un acercamiento al trabajo de enseñanza que los profesores universitarios realizamos desde una aproximación a los textos escritos como producciones "enunciadas por alguien y destinadas a alguien". De diferentes formas, el diálogo, como metáfora del entramado discursivo que forma parte del conocimiento académico y de las literacidades asociadas al mundo universitario, está presente en los tres metaeventos. La presencia del diálogo se manifiesta con claridad en el último, no solo en el reconocimiento de los ecos de algo dicho antes en esa construcción, sino en la afirmación de que los docentes universitarios no empezamos "de cero" en cada encuentro con nuestros estudiantes. Pero el diálogo también está presente en el primer metaevento cuando al pensar sobre cómo usar dos distintos manuales se plantea la posibilidad de aproximarse a sus lecturas estableciendo interlocuciones entre ambos manuales - enunciados (interlocuciones que pueden ser de acuerdo, o no). Y, si bien en el segundo metaevento el centro de atención no se puso allí, recuerdo al lector de este trabajo que la actividad práctica revisada incorpora dos textos para trabajar en clase (cuestión que forma parte de la dinámica regular de los profesores de ese curso), en la búsqueda de un pretendido establecimiento de diálogo entre los textos seleccionados.

Si nos apropiamos de los usos del lenguaje en contacto con ellos, si los sentidos nacen del encuentro entre enunciados y Discursos pasados y futuros en formas dialógicas variadas, el ámbito académico no es la excepción. Allí son muchos y muy diversos los Discursos, y múltiples los enunciados que debemos aprender a escuchar (o leer). Si como profesores universitarios, al enseñar a nuestros estudiantes partimos desde un lugar dialógico, nuestras acciones didácticas se verían enriquecidas al traer la sofisticación de los órdenes discursivos (FAIRCLOUGH, 2005) por los que discurren las literacidades académicas. Al fin y al cabo, no se trata de "leer y escribir textos" a secas, sino de "enunciar según el género (discursivo)" dentro de un dominio 
institucional socialmente complejo.

El abordaje desde una pedagogía de las multiliteracidades de la enseñanza universitaria puede ser un camino futuro en cuanto a las acciones didácticas (que de una u otra forma involucrarán la lectura y la escritura) en distintos ámbitos disciplinares. Frente a la postura que propone "aislar" el conocimiento de la lectura y la escritura del propio conocimiento disciplinar (mediante la oferta de talleres por fuera de los cursos disciplinares), se abre la posibilidad de trabajar la lectura y la escritura desde el conocimiento situado (por tanto, disciplinar). Claro que, este camino puede recorrerse siempre y cuando los docentes a cargo de los cursos disciplinares estemos dispuestos a asumir, como intenté mostrar desde los distintos metaeventos, que la lectura y la escritura en las diferentes disciplinas no es un tema meramente contenidístico, sino discursivo (con todo lo asociado al lenguaje que esto conlleva).

Las prácticas letradas son, al mismo tiempo, prácticas discursivas y prácticas sociales. En tanto que prácticas discursivas, articulan determinados usos lingüísticos de los participantes (sujetos con creencias, actitudes, historia) con sus formas de relacionarse socialmente y con el mundo material en el que se sitúan, y son esos usos lingüísticos los que se configuran como órdenes discursivos propios de las diversas prácticas. Muchas veces las prácticas -discursivas, sociales, letradas- orientan lo acontecido en los eventos, pero esto no se da de forma unidireccional. El juego entre eventos (letrados, en este caso) y prácticas (letradas), es decir entre situaciones concretas y praxis sociocultural es permanente, porque los participantes en interacciones situadas contribuyen a través del tiempo al sustento o al cambio de las prácticas (LINELL, 2009). Si los profesores universitarios nos acercamos a la enseñanza desde criterios situados, con instrucciones explícitas y encuadre crítico de nuestras prácticas, podemos apostar a que las perspectivas presentes nos conduzcan a horizontes futuros de nuestras prácticas, transformadas y mejores.

\section{Referencias}

BAJTíN, M. M. (1952-53) El problema de los géneros discursivos. In: BAJTíN, M. M. Estética de la creación verbal. Traducido por T. Bubnova. Buenos Aires: Siglo veintiuno editores, 2002. p. 248-293.

BAJTín, M. M. (1959-61) El problema del texto en la lingüística, la filología y otras ciencias humanas. In: BAJTín, M. M. Estética de la creación verbal. Traducido por T. Bubnova. Buenos Aires: Siglo veintiuno editores, 2002. p. 294-323.

BAJTín, M. M. (1961) Para una reelaboración del libro sobre Dostoievski. In: BAJTíN, M. M. Estética de la creación verbal. Traducido por T. Bubnova. Buenos Aires: Siglo veintiuno editores, 2002. p. 324-345.

BARTON, D. Literacy: an introduction to the ecology of written language. Oxford: Blackwell, 1994. 
BAZERMAN, Ch. El descubrimiento de la escritura académica. In: NAVARRO, F. (coord.) Manual de escritura para carreras de humanidades. Buenos Aires: Editorial de la Facultad de Filosofía y Letras, Universidad de Buenos Aires, 2014. p. 11-16.

BRAIT, B. Análise e teoria do discurso. In: BRAIT, B. (org.) Bakhtin: outros conceitos- chave. São Paulo: Contexto, 2006. p. 95-114.

CARLINO, P. Escribir, leer y aprender en la universidad. Buenos Aires: Fondo de Cultura Económica, 2007.

FAIRCLOUGH, N. Multiliteracies and language: orders of discourse and intertextuality. In: KALANTZIS, M.; COPE, B. (Eds.) Multiliteracies: literacy learning and the design of social futures. London and New York: Routledge, 2005. p. 162-181.

FARACO, C. A. Linguagem \& diálogo: as ideias linguísticas do círculo de Bakhtin. 2. ed. Paraná, Criar, 2006.

GARCÍA DE LOS SANTOS, E.; MÉNDEZ CAULÍN, M. Las consignas como reguladoras de la lectura y la escritura: la experiencia de los talleres del Programa LEA. In: BERTOLOTTI, V. (comp.) et. al. Lengua, comunicación e información. Montevideo: Facultad de Información y Comunicación, 2018. p. 142-154.

GEE, J. P. La ideología en los discursos: lingüística social y alfabetizaciones. Madrid - A Coruña: Eds. Morata y Fundación Paideia Galiza, 2005.

GEE, J. P. Discourse and sociocultural studies in reading. In: KAMIL, M. L. et al. (eds.) Handbook of Reading Research: volume III. New Jersey, London: Lawrence Erlbaum, 2000a. p. 195-207.

HYLAND, K. Disciplinary discourses: social interactions in academic writing. USA: The University of Michigan Press, 2004.

KALANTZIS, M.; COPE, B. A multiliteracies pedagogy: a pedagogical supplement. In: KALANTZIS, M.; COPE, B. (Eds.) Multiliteracies: literacy learning and the design of social futures. London and New York: Routledge, 2005. p. 239-248.

LEA, M. R.; STREET, B. Writing as academic literacies: understanding textual practices in higher education. In: CANDLIN, C. N.; HYLAND, K. (Eds.) Writing: texts, processes and practices. United Kingdom: Longman, 1999. p. 62-81.

LILLIS, T. M. Student writing: access, regulation, desire. London and New York: Routledge, 2001. https://doi.org/10.4324/9780203186268

LINELL, P. Rethinking language, mind and world dialogically: interactional and contextual theories of human sense-making. USA: Information Age, 2009.

NARVAJA DE ARNOUX, E.; DI STEFANO, M.; PEREIRA, C. La lectura y la escritura en la universidad. Buenos Aires: Eudeba, 2006.

NATALE, L.; STAGNARO, D. El parcial presencial. In: NAVARRO, F. (coord.) Manual de escritura para carreras de humanidades. Buenos Aires: Editorial de la Facultad de Filosofía y Letras, Universidad de Buenos Aires, 2014. p. 102-134.

SCARDAMALIA, M.; BEREITER, C. Dos modelos explicativos de los procesos de composición $\begin{array}{llllll}\text { escrita. Infancia y } & \text { Aprendizaje, } & \text { 58, }\end{array}$ https://doi.org/10.1080/02103702.1992.10822332

SOARES, M. Letramento: um tema em três gêneros. 2a․ ed., 11a reimpr. Belo Horizonte: 
Autêntica, 2006.

SOBRAL, A. Do dialogismo ao gênero: as bases do pensamento do círculo de Bakhtin. Campinas: Mercado de Letras, 2009.

STREET, B. V. What's "new" in New Literacy Studies? Current Issues in Comparative Education, Vol 5 (2). p. 77-91, 2003.

STREET, B. V. Social Literacies: Critical Approaches to Literacy in Development, Ethnography and Education. London and New York: Longman, 1995.

STREET, B. V. The new literacy studies, guest editorial. Journal of Research in Reading, United Kingdom, U.S.A., 16(2), p. 81-97, 1993. https://doi.org/10.1111/j.1467-9817.1993.tb00039.x

VÁZQUEZ, A. "Consignas de escritura: entre la palabra del docente y los significados de los estudiantes". Cuadernillos de actualización para pensar la enseñanza universitaria, Universidad de Río Cuarto, n. 2, v. 7. p. 4-16, 2007.

VOLÓSHINOV, V. N. El marxismo y la filosofía del lenguaje. Traducido por T. Bubnova. Buenos Aires: Ediciones Godot, Colección Exhumaciones, 2009.

Recebido em: 08/04/2019

Aceito em: 12/06/2019 